\title{
The Inconsistency Problem of Riemann Zeta Function Equation
}

\section{Mei Xiaochun}

Department of Theoretic Physics and Pure Mathematics, Institute of Innovative Physics in Fuzhou, Fuzhou, China

Email address:

ycwlyjs@yeah.net,mxc001@163.com

\section{To cite this article:}

Mei Xiaochun. The Inconsistency Problem of Riemann Zeta Function Equation. Mathematics Letters. Vol. 5, No. 2, 2019 , pp. 13-22. doi: $10.11648 /$ j.ml.20190502.11

Received: July 8, 2019; Accepted: July 31, 2019; Published: August 13, 2019

\begin{abstract}
Four basic problems in Riemann's original paper are found. The Riemann hypothesis becomes meaningless. 1. It is proved that on the real axis of complex plane, the Riemann Zeta function equation holds only at point $\operatorname{Re}(\mathrm{s})=1 / 2(\mathrm{~s}=\mathrm{a}+\mathrm{ib})$. However, at this point, the Zeta function is infinite, rather than zero. At other points of real axis, the two sides of Zeta function equation are contradictory. When one side is finite, another side may be infinite. 2. An integral item around the original point of coordinate system was neglected when Riemann deduced the integral form of Zeta function. The item was convergent when $\operatorname{Re}(s)>1$ but divergent when $\operatorname{Re}(s)<1$. The integral form of Zeta function does not change the divergence of its series form. Two reasons to cause inconsistency and infinite are analyzed. 3. When the integral form of Zeta function was deduced, a summation formula was used. The applicable condition of this formula is $\mathrm{x}>0$. At point $\mathrm{x}=0$, the formula is meaningless. However, the lower limit of Zeta function integral is $\mathrm{x}=0$, so the formula can not be used. 4. A formula of Jacobi function was used to prove the symmetry of Zeta function equation. The applicable condition of this formula was also $\mathrm{x}>0$. However, the lower limit of integral in the deduction was $\mathrm{x}=0$. So this formula can not be used too. The zero calculation of Riemann Zeta function is discussed at last. It is pointed out that because approximate methods are used, they are not the real zeros of strict Riemann Zeta function.
\end{abstract}

Keywords: Riemann Hypothesis, Riemann Zeta Function, Zeta Function Equation, Jacobi’s Function, Residue Theorem, Cauchy-Riemann Equation

\section{Introduction}

Riemann Zeta function is an important one in modern mathematics. Riemann proposed the Riemann hypothesis about the zeros of Zeta function in 1859 , but it can not be proved up to now. In this is paper, the Riemann's original deduction is examined carefully and four basic mistakes are revealed. Due to theses mistakes, the integral form of Riemann Zeta function and its function equation can not hold. Riemann hypothesis becomes meaningless.

Riemann Zeta function has two forms. One is the form of series summation and another is the form of integral. The series summation form is original and can be written as

$$
\zeta(s)=\sum_{n=1}^{\infty} n^{-s}=1+\frac{1}{2^{s}}+\frac{1}{3^{s}}+\frac{1}{4^{s}}+\cdots
$$

Here $s=a+i b \in C$ is a complex number, $a \in R$ and $b \in R$ are real numbers. If $S$ is a real number with $b=0$ and $a>1$, the series is convergent, while $b=0$ and $a \leq 1$, the series is divergent. For example

$$
\begin{gathered}
\zeta(1)=1+\frac{1}{2}+\frac{1}{3}+\frac{1}{4}+\cdots \rightarrow \infty \\
\zeta(2)=1+\frac{1}{2^{2}}+\frac{1}{3^{2}}+\frac{1}{4^{3}}+\cdots=\frac{\pi^{2}}{6} \\
\zeta(-2)=1+2^{2}+3^{2}+4^{2}+\cdots \rightarrow \infty
\end{gathered}
$$

Here $\zeta(1)$ is the harmonic series and $\zeta(2)$ is the Euler formula. If $s$ is a complex number, according to general understanding, when $\operatorname{Re}(s)>1$, (1) is convergent. While $\operatorname{Re}(s) \leq 1,(1)$ is divergent and meaningless.

In order to make (1) meaningful for the situation $\operatorname{Re}(s)<1$, Riemann transformed it into the integral form by means of the Gama function and obtained $[1,2]$ 


$$
\left(\sum_{n=1}^{\infty} n^{-s}\right) \Gamma(s)=\int_{0}^{\infty} \frac{x^{s-1}}{e^{x}-1} d x \quad \operatorname{Re}(s)>1
$$

Here $x \in R$ is still a real number. In order to calculate (3), Riemann extended it to the integral of complex plane with $(z \in C)$.

$$
\sum_{n=1}^{\infty} n^{-s}=\frac{\Gamma(1-s)}{2 \pi i} \int_{K} \frac{(-z)^{s-1}}{e^{z}-1} d z \quad \operatorname{Re}(s) \neq 1
$$

By means of the method of residues and based on (4), Riemann obtained following Zeta function equation

$$
\sum_{n=1}^{\infty} n^{-s}=2(2 \pi)^{s-1} \Gamma(1-s) \sin \left(\frac{s \pi}{2}\right) \sum_{n=1}^{\infty} n^{-(1-s)}
$$

By using the definition of (1), (5) was written as in general

$$
\zeta(s)=2(2 \pi)^{s-1} \Gamma(1-s) \sin \left(\frac{s \pi}{2}\right) \zeta(1-s)
$$

According to common understanding, after the complex continuation, (6) was tenable on whole complex plane except at the point $\operatorname{Re}(s)=1$ [2]. Then Riemann introduced following transformation $[1,3]$

$$
\xi(s)=\frac{1}{2} s(s-1) \pi^{-s / 2} \Gamma\left(\frac{s}{2}\right) \zeta(s)
$$

and proved the existence of symmetry

$$
\xi(s)=\xi(1-s)
$$

Because $\zeta(s)$ and $\xi(s)$ were considered to have the same zeros, the zero's calculation of Zeta function equation was based on (7) actually.

According to (6), when $s= \pm 2 k \quad(k=0,1,2,3, \cdots)$, $\zeta(s)=0$. For the situations with $s=-2 k$, the zeros were called as trivial ones $[3,4]$. The situations with $s=+2 k$ were not discussed in general.

Because the distribution of prime numbers is related to the zeros of Zeta function, the judgment of zeros becomes an impotent problem. Riemann hypothesis declared that all zeros were distributed on the straight line $\operatorname{Re}(s)=1 / 2$ of complex plane. But the hypothesis can not be proved up to now $[5,6]$.

The original paper of Riemann is analyzed in this paper. Four basic mistakes are found which makes Riemann hypothesis meaningless. They are:

1. There exists inconsistency in the Riemann Zeta function equation. After complex continuation, (5) and (6) were considered to be tenable on whole complex plane except the point $\operatorname{Re}(s)=1$. However, this is not true.

So-called continuation of function indicates to re-define the function in a new domain where the original function has no definition so that the function becomes meaningful. Therefore, there is a basic principle for the continuation of function. At each point of original domain, re-defined function should have the same value with original function, though re-defined function may have different form in new domain. Otherwise, it can not be regarded as the continuation of original function.

According to this principle, on the domain of $\operatorname{Re}(s)>1$, the left side of (6) should be equal to (1). The right sides of (5) and (6) have definition except at point $\operatorname{Re}(s)=1$, i.e., $a \neq 1$ but $b$ can be arbitrary. The domain of left sides of (5) and (6) is $\operatorname{Re}(s)>1$, i.e., $a>1$ but $b$ can be arbitrary. Therefore, to take $a=3.5$ and $b=0$, (5) and (6) should be effective. However, the result of practical calculation is that the left side of (5) is a finite value, but the right side of (5) is infinite. Therefore, two sides of Zeta function equation are incompatible. (5) and (6) do not hold.

In fact, it is proved that on the real axis of complex plane, (5) only hold at point $a=1 / 2$. But in this case, two sides of Zeta function equation are equal to infinite, rather than zero. That is to say, on the real axis, the point $a=1 / 2$ is the infinite point of Riemann Zeta function equation, rather than zero.

When $a$ is a non-integer with $|a|>1$, if the left side of (5) is convergent, its right side is infinite, vice versa. When $0<a<1$, the two sides of (5) are infinite and become meaningless. So the Riemann Zeta function equation is contradictory.

2. An integral item around the original point of coordinate system was neglected in Riemann's original paper. The item was convergent when $\operatorname{Re}(s)>1$, but infinite when $\operatorname{Re}(s) \leq 1$. That is to say, the complex continuation of Riemann Zeta function has not changed its divergence of series summation form.

Two reasons to cause the inconsistency and divergence are analyzed. They are

3. A summation formula was used in Riemann's deduction. The applicable condition of this formula is $x>0$. At point $x=0$, the formula is meaningless. However, the lower limit of Zeta function integral is $x=0$. So this formula can not be used.

4. The formula $\theta(x)=\sqrt{x} \theta(1 / x)$ of Jacobi function was used to prove the symmetry of Zeta function. The applicable condition of this formula is also $x>0$ [7]. However, the lower limit of integral involved in the deduction is $x=0$. Therefore, the formula can not be used too, the symmetry $\xi(s)=\xi(1-s)$ does not hold.

The zero calculations of Riemann Zeta function was discussed at last. It was pointed out that because approximate method was used, the Cauchy-Riemann equation was not satisfied. Thought great numbers of zeros were founded on the straight line $\operatorname{Re}(s)=1 / 2$ of complex plane, they were not real zeros of strict Zeta function.

\section{The Deduction of Riemann Zeta Function Equation}

\subsection{The Deduction of Integral Form of Riemann Zeta Function}

Based on the form of series summation and by using Gama 
function, Riemann deduced the integral form of $\zeta(s)$ function $[1,2]$. The definition of Gama function is

$$
\Gamma(s)=\int_{0}^{\infty} e^{-x} x^{s-1} d x \quad \operatorname{Re}(s)>0
$$

Here $x \in R$ is a real number and $s=a+i b \in C$ is a complex number with $\operatorname{Re}(s)>0$. Let $x \rightarrow n x$, (9) becomes

$$
\begin{aligned}
& \Gamma(s)=\int_{0}^{\infty} e^{-(n x)}(n x)^{s-1} d(n x)=n^{s} \int_{0}^{\infty} e^{-n x} x^{s-1} d x \\
& \text { or } \quad\left(\sum_{n=1}^{\infty} n^{-s}\right) \Gamma(s)=\int_{0}^{\infty}\left(\sum_{n=1}^{\infty} e^{-n x}\right) x^{s-1} d x
\end{aligned}
$$

Then, Riemann used the following summation formula of series (The original paper of Riemann had not provided this formula but used it actually.) [8].

$$
\begin{gathered}
\sum_{n=1}^{\infty} e^{-n x}=e^{-x}+e^{-2 x}+e^{-3 x}+\cdots \\
=e^{-x}\left(1+e^{-x}+e^{-2 x}+e^{-3 x}+\cdots\right) \\
=\frac{e^{-x}}{1-e^{-x}}=\frac{1}{e^{x}-1}
\end{gathered}
$$

By substituting (12) in (11), Riemann obtained

$$
\left(\sum_{n=1}^{\infty} n^{-s}\right) \Gamma(s)=\int_{0}^{\infty} \frac{x^{s-1}}{e^{x}-1} d x \quad \operatorname{Re}(s)>1
$$

In order to calculate (13), Riemann extended the integral of real number $x$ into complex number $z=x+i y \in C$ by defining the function $I(z)$ with

$$
I(s)=\int_{L} \frac{(-z)^{s-1}}{e^{z}-1} d z \quad \operatorname{Re}(s) \neq 1
$$

The definition domain of function was extended to whole complex plane except the point $s=1$. As shown in Figure 1, the path of integral started from $x \rightarrow \infty$ to $x=\delta$ along the straight line $B$ under $x$ axis. Here $\delta$ was a small quantity. Then path was along the circle $\Omega$ with radius $\sqrt{x^{2}+y^{2}}=\delta$ around the original point of the coordinate system. At last, the path was from $x=\delta$ to $x \rightarrow \infty$ along the straight line $A$ above $x$ axis.

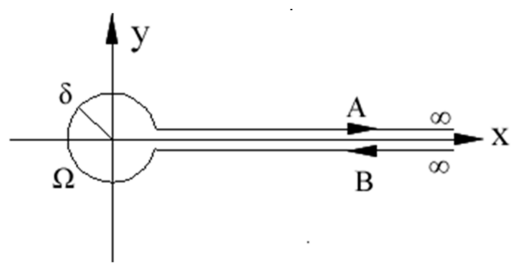

Figure 1. The integral path of Riemann Zeta function.
According to Figure 1, (14) contained three items

$$
I(s)=\int_{\infty}^{\delta} \frac{(-x)^{s-1}}{e^{x}-1} d x+\int_{\Omega} \frac{(-z)^{s-1}}{e^{z}-1} d z+\int_{\delta}^{\infty} \frac{(-x)^{s-1}}{e^{x}-1} d x
$$

Riemann's paper provided following result directly without concrete calculation $[1,2]$

$$
I(s)=\left(e^{i \pi s}-e^{-i \pi s}\right) \int_{0}^{\infty} \frac{x^{s-1}}{e^{x}-1} d x
$$

It indicated that Riemann assumed that the medium item on the right side of (15) was zero with

$$
\int_{\Omega} \frac{(-z)^{s-1}}{e^{z}-1} d z=0
$$

By using the Euler's formula

$$
\frac{e^{i \pi s}-e^{-i \pi s}}{2 i}=\sin (\pi s)
$$

and substituting (16) and (18) in (13), the result was

$$
I(s)=i 2 \sin (\pi s) \Gamma(s)\left(\sum_{n=1}^{\infty} n^{-s}\right)
$$

By using the complementary formula of Gama function

$$
\Gamma(s) \Gamma(1-s)=\frac{\pi}{\sin (\pi s)}
$$

and considering (14), (19) can be written as

$$
\sum_{n=1}^{\infty} n^{-s}=\frac{I(s)}{i 2 \sin (\pi s) \Gamma(s)}=\frac{\Gamma(1-s)}{2 \pi i} \int_{L} \frac{(-z)^{s-1}}{e^{z}-1} d z
$$

By using the definition (1), (21) is generally written as

$$
\zeta(s)=\frac{I(s)}{i 2 \sin (\pi s) \Gamma(s)}=\frac{\Gamma(1-s)}{2 \pi i} \int_{L} \frac{(-z)^{s-1}}{e^{z}-1} d z
$$

In this way, Riemann deduced the integral form of Zeta function. However, according to (21) we should note that the Zeta function $\zeta(s)$ shown in (22) is in the form of (1). This is very impotent for us to discuss the consistency problem of Zeta function equation.

\subsection{The Deduction of Riemann Zeta Function Equation}

In order to calculate the right side of (22), Riemann used the Residue theorem by considering the contour shown in Figure 2 . The integrand function has infinite poles on the points $z= \pm i 2 n \pi \quad(n=1,2,3, \cdots)$, but only three are drawn. The path of integral in Figure 1 is enlarged into a closed curve $C$, composed of a big circle $C_{0}$ and the paths shown in Figure 1. 
According to the Residue theorem, when the radius of $C$ tends to infinite, the integral along big circle $C_{0}$ is zero.

The calculation formula of Residue integral is [9]

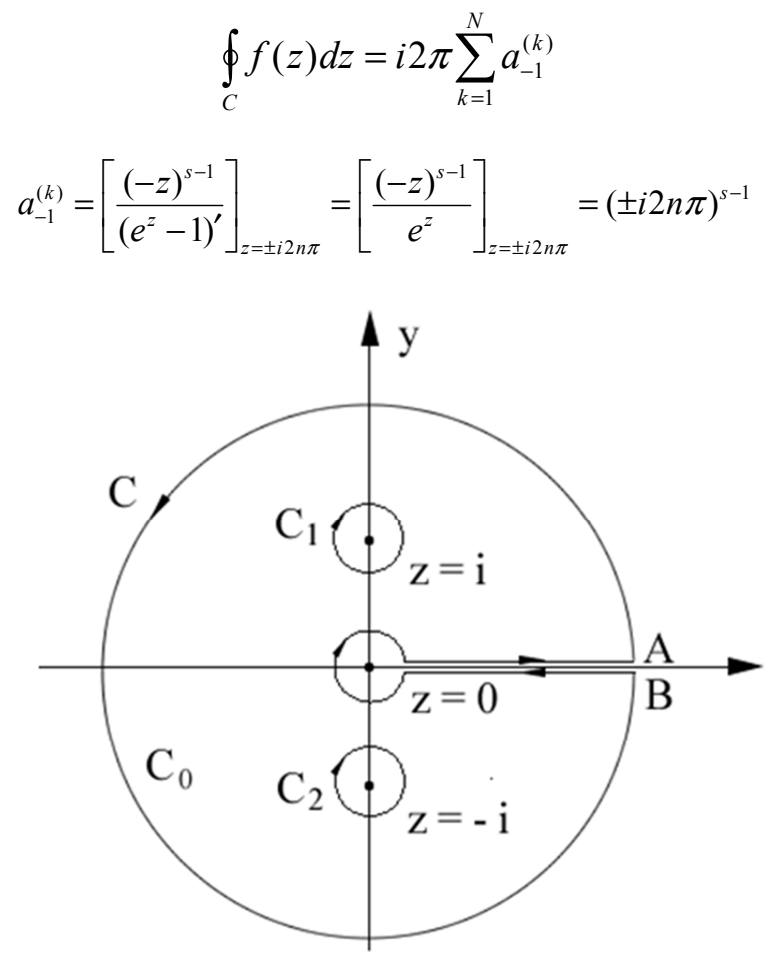

Figure 2. The residual integral of Riemann Zeta function.

By considering

$$
(i)^{s-1}+(-i)^{s-1}=\frac{1}{i}\left(e^{i \pi s / 2}-e^{-i \pi s / 2}\right)=2 \sin \frac{\pi s}{2}
$$

the calculation result of $(23)$ is

$$
\begin{gathered}
\sum_{k=1}^{N} a_{-1}^{(k)}=\frac{1}{i 2 \pi} \int_{C} \frac{(-z)^{s-1}}{e^{z}-1} d z=\sum_{n=1}^{\infty}\left[(i 2 \pi n)^{s-1}+(-i 2 \pi n)^{s-1}\right] \\
=2(2 \pi)^{s-1} \sin \frac{\pi s}{2} \sum_{n=1}^{\infty} n^{-(1-s)}
\end{gathered}
$$

Substituting (26) in (21), we have

$$
\sum_{n=1}^{\infty} n^{-s}=2(2 \pi)^{s-1} \Gamma(1-s) \sin \frac{\pi s}{2} \sum_{n=1}^{\infty} n^{-(1-s)}
$$

In light of the definition of (1), we have

$$
\sum_{n=1}^{\infty} n^{-(1-s)}=\zeta(1-s)
$$

So (27) can be written as

$$
\zeta(s)=2(2 \pi)^{s-1} \Gamma(1-s) \sin \frac{\pi s}{2} \zeta(1-s)
$$

(29) is just the Riemann Zeta function equation, or called as the algebraic relation of Zeta function. Based on it, Riemann proposed the Riemann hypothesis that all zeros of Zeta functions were located on the straight line of $s=1 / 2+i b$ on the complex plane.

At present, the common understanding is that through the complex continuation, (29) becomes the new definition of Riemann Zeta function. The function $\zeta(s)$ on the left hand side of (29) is defined by the right side, which is not the original form defined in (1) again.

However, this is not the case. When we deduce (29), (1) and (28) must be used finally. The prototype of (29) is (27). (29) is only a simplified notation of (27). So, we should discuss the consistency problem of the Riemann Zeta function equation based on (27).

\section{The Proof That Zeta Function Equation Does not Hold on the Real Axis of Complex Plane}

According to the theory of complex variable function, the complex continuation indicates that a real function $f(x)$ is extended to the field of complex number with $f(x) \rightarrow G(z)=G(x+i y)$. Thought the definition domain of function is changed, on the real axis with $y=0$, the complex continuation function should be the same as the original function with $G(x)=f(x)$. If they are not the same on the real axis, the complex continuation of function can not be considered correct.

Therefore, (27) should hold on the real axis of complex plane with $b=0$ and $a \neq 1$. Because the definitions of Zeta function $\zeta(s)$ and $\zeta(1-s)$ shown in (1) and (28) are certain, on the real axis of complex plane, (27) becomes

$$
\sum_{n=1}^{\infty} n^{-a}=2(2 \pi)^{a-1} \Gamma(1-a) \sin \frac{a \pi}{2} \sum_{n=1}^{\infty} n^{a-1}
$$

However, it can be proved that the two sides of (30) are contradictory, so (30) can not hold.

I) When $a$ is an integer, for example, to take $a=-2$, the left side of (30) is infinite with

$$
\sum_{n=1}^{\infty} n^{2}=1+2^{2}+3^{2}+4^{2}+\cdots \rightarrow \infty
$$

But the right side of (30) is zero with

$$
\begin{aligned}
& 2(2 \pi)^{-3} \Gamma(3) \sin (-\pi) \sum_{n=1}^{\infty} n^{-3} \\
= & 0 \times\left(1+\frac{1}{2^{3}}+\frac{1}{3^{3}}+\frac{1}{4^{3}}+\cdots\right)=0
\end{aligned}
$$

To take $a=4$, the left side of (30) is a limited and certain value with 


$$
\sum_{n=1}^{\infty} n^{-4}=1+\frac{1}{2^{4}}+\frac{1}{3^{4}}+\frac{1}{4^{4}}+\cdots>1
$$

But the right side of (30) is uncertain with

$$
\begin{gathered}
2(2 \pi)^{3} \Gamma(-3) \sin (2 \pi) \sum_{n=1}^{\infty} n^{3} \\
=0 \times\left(1+2^{3}+3^{3}+4^{3}+\cdots\right)=0 \times \infty
\end{gathered}
$$

The Zeta functions on the two sides of (30) are incompatible.

II) When $a$ is a non-integer, to take $a=1 / 2$, called as the non-trivial zero of Riemann hypothesis, the left side of (30) is

$$
\begin{gathered}
\sum_{n=1}^{\infty} n^{-1 / 2}=1+\frac{1}{\sqrt{2}}+\frac{1}{\sqrt{3}}+\frac{1}{\sqrt{4}}+\cdots \\
>1+\frac{1}{2}+\frac{1}{3}+\frac{1}{4}+\cdots \rightarrow \infty
\end{gathered}
$$

Due to $\Gamma(1 / 2)=\sqrt{\pi}$ and $\sin \pi / 4=\sqrt{2} / 2$, the right side of (30) is

$$
\begin{gathered}
\frac{2}{\sqrt{2 \pi}} \Gamma(1 / 2) \sin \frac{\pi}{4} \sum_{n=1}^{\infty} n^{-1 / 2} \\
=1 \times\left(1+\frac{1}{\sqrt{2}}+\frac{1}{\sqrt{3}}+\frac{1}{\sqrt{4}}+\cdots\right) \rightarrow \infty
\end{gathered}
$$

The two sides of (30) are completely identical, but they are infinite, rather than zero. So $a=1 / 2$ is not the zero of Riemann Zeta function equation on the real axis of complex plane.

III) If $a>1$ is a non-integer, for example, to take $a=3.5$, the left side of (30) is convergent with

$$
\begin{aligned}
\sum_{n=1}^{\infty} n^{-a} & =1+\frac{1}{2^{3.5}}+\frac{1}{3^{3.5}}+\frac{1}{4^{3.5}}+\cdots \\
& <1+\frac{1}{2}+\frac{1}{3}+\frac{1}{4}+\cdots
\end{aligned}
$$

But the right side of (30) is divergent. According the formula of negative continuation of Gama function with $\Gamma(-2.5)=-8 \sqrt{\pi} / 15$, as well as $\sin 3.5 \pi / 2=-0.7091$, we have

$$
2(2 \pi)^{a-1} \Gamma(1-a) \sin \frac{a \pi}{2} \sum_{n=1}^{\infty} n^{a-1}
$$

$$
\begin{gathered}
=2(2 \pi)^{2.5} \Gamma(-2.5) \sin \frac{3.5 \pi}{2} \sum_{n=1}^{\infty} n^{2.5} \\
=(2 \pi)^{2.5} \sqrt{\pi} \times 0.7564 \\
\times\left(1+2^{2.5}+3^{2.5}+4^{2.5}+\cdots\right) \rightarrow \infty
\end{gathered}
$$

IV) If $a<0$ is a non-integer, for example, to take $a=-3.5$, the situation is conversed. The left side of (30) is divergent with

$$
\sum_{n=1}^{\infty} n^{-a}=1+2^{3.5}+3^{3.5}+4^{3.5}+\cdots \rightarrow \infty
$$

But the right side of (30) is convergent. Due to $\Gamma(4.5)=105 \sqrt{\pi} / 16$ and $\sin (-3.5 \pi / 2)=0.7091$, we have

$$
\begin{gathered}
2(2 \pi)^{a-1} \Gamma(1-a) \sin \frac{a \pi}{2} \sum_{n=1}^{\infty} n^{a-1} \\
=2(2 \pi)^{-4.5} \Gamma(4.5) \sin \left(\frac{-3.5 \pi}{2}\right) \sum_{n=1}^{\infty} n^{-4.5} \\
=(2 \pi)^{-4.5} \sqrt{\pi} \times 9.3069 \\
\times\left(1+\frac{1}{2^{4.5}}+\frac{1}{3^{4.5}}+\frac{1}{4^{4.5}}+\cdots\right)
\end{gathered}
$$

V) If $0<a<1$, for example, to take $a=0.2$, the left side of (30) is divergent with

$$
\begin{gathered}
\sum_{n=1}^{\infty} n^{-a}=1+\frac{1}{2^{0.2}}+\frac{1}{3^{0.2}}+\frac{1}{4^{0.2}}+\cdots \\
>1+\frac{1}{2}+\frac{1}{3}+\frac{1}{4}+\cdots \rightarrow \infty
\end{gathered}
$$

The right side of (30) is also divergent with

$$
\begin{gathered}
2(2 \pi)^{a-1} \Gamma(1-a) \sin \frac{a \pi}{2} \sum_{n=1}^{\infty} n^{a-1} \\
=2(2 \pi)^{-0.8} \Gamma(0.8) \sin (0.1 \pi) \times \sum_{n=1}^{\infty} n^{-0.8} \\
=(2 \pi)^{-4.5} \times 0.7188 \\
\times\left(1+\frac{1}{2^{0.8}}+\frac{1}{3^{0.8}}+\frac{1}{4^{0.8}}+\cdots\right) \rightarrow \infty
\end{gathered}
$$

Here $\Gamma(0.8)=\Gamma(1.8) / 0.8=1.1643, \sin (0.1 \pi)=0.3087$. In this case, (30) is meaningless. Different from (35) and (36), we can not ensure that the two sides of equal sign are certainly equal. 
In short, when $a$ is an non-integer with $|a|>1$, the Riemann Zeta function equation is inconsistent on the real axis of complex plane. If one side of equation is infinite, another side is limited. When $0<a<1$, the two sides of equation are infinite. We can not ensure that two sides are certainly equal. Only at the point $a=1 / 2$, the two sides of equation are equal strictly. But they are infinite, rather than zeros. That is to say, $s=a=1 / 2$ is not the zero of Riemann Zeta function equation, it is the point of infinite. This result is different from the Riemann hypothesis.

\section{The Non-Negligible Item in the Integral Form of Zeta Function}

\subsection{Formula (17) Is Not Equal to Zero When $\operatorname{Re}(s)<1$}

We now prove that the integral (17) is divergent rather than zero when $\operatorname{Re}(s)<1$. Let $z=\delta e^{i \theta}$ describe the path around the small circle $\Omega$, we have $d z=i \delta e^{i \theta} d \theta, d z / z=i d \theta$. When $\delta<<1$, we have

$$
\begin{gathered}
\frac{1}{e^{z}-1}=\frac{1}{1+z+z^{2} / 2 !+z^{3} / 3 !+\cdots-1} \\
=\frac{1}{z\left(1+z / 2 !+z^{2} / 3 !+\cdots\right)}=\frac{1}{z}\left(1+k_{1} z+k_{2} z^{2}+\cdots\right)
\end{gathered}
$$

Here $k_{i}$ are the developing parameters. Substituting (43) in the left side of (17), we obtain

$$
\int_{\Omega} \frac{(-z)^{s-1}}{e^{z}-1} d z=\int_{\Omega} \frac{(-z)^{s-1}}{z}\left(1+k_{1} z+k_{2} z^{2}+\cdots\right) d z
$$

Let's only calculate the first item with

$$
\begin{gathered}
\int_{\Omega} \frac{(-z)^{s-1}}{z} d z=\int_{0}^{2 \pi}\left(-\delta e^{i \theta}\right)^{s-1} i d \theta \\
=(-\delta)^{s-1} \int_{0}^{2 \pi} e^{i(s-1) \theta} d(i \theta)=\left.\frac{(-\delta)^{s-1}}{s-1} e^{i(s-1) \theta}\right|_{0} ^{2 \pi} \\
=\frac{(-\delta)^{s-1}}{s-1}\left(e^{i 2 \pi s} e^{-i 2 \pi}-1\right)=\frac{(-\delta)^{s-1}}{s-1}\left(e^{i 2 \pi s}-1\right)
\end{gathered}
$$

Substituting $s=a+i b$ in (45) and considering

$$
e^{i 2 \pi s}=e^{-2 \pi b} e^{i 2 \pi a}=e^{-2 \pi b}(\cos 2 \pi a+i \sin 2 \pi a)
$$

and $\delta^{\mathrm{i} b}=e^{i b \ln \delta}$, we get

$$
\frac{(-\delta)^{s-1}}{s-1}=\frac{\left(\delta e^{i \pi}\right)^{a-1+i b}}{a-1+i b}=\frac{\delta^{a-1} \delta^{i b} e^{-\pi b} e^{i \pi(a-1)}}{a-1+i b}
$$

$$
\begin{gathered}
=\frac{\delta^{a-1} e^{-\pi b} e^{i b \ln \delta} e^{i \pi(a-1)}}{(a-1)^{2}+b^{2}}(a-1-i b) \\
=\frac{\delta^{a-1} e^{-\pi b}}{(a-1)^{2}+b^{2}}\{(a-1) \cos (b \ln \delta+\pi(a-1)) \\
+b \sin (b \ln \delta+\pi(a-1)) \\
+i[(a-1) \sin (b \ln \delta+\pi(a-1)) \\
-b \cos (b \ln \delta+\pi(a-1))]\}
\end{gathered}
$$

When $\delta \rightarrow 0$ we have $\ln \delta \rightarrow-\infty, \quad \sin (b \ln \delta)$ and $\cos (b \ln \delta)$ are uncertain. If $a>1$, when $\delta \rightarrow 0, \delta^{a-1} \rightarrow 0$, so (46) is convergent. If $a<1$, when $\delta \rightarrow 0, \delta^{a-1} \rightarrow \infty$. In this case, (46) is infinite and (44) is divergent too.

The calculation result of second item in (44) is

$$
\begin{gathered}
k_{1} \int_{\Omega}(-z)^{s-1} d z=k_{1} \int_{0}^{2 \pi}\left(-\delta e^{i \theta}\right)^{s-1} \delta d e^{i \theta} \\
=k_{1}\left(e^{i \pi}\right)^{s-1} \delta^{s} \int_{0}^{2 \pi} e^{i s \theta} d(i \theta)=\left.\frac{k_{1} e^{i \pi(\mathrm{s}-1)} \delta^{s}}{s} e^{i s \theta}\right|_{0} ^{2 \pi} \\
=\frac{k_{1} e^{i \pi(\mathrm{s}-1)} \delta^{s}}{a^{2}+b^{2}}\left(e^{i 2 \pi s}-1\right)(a-i b) \sim k_{1}^{\prime} \delta^{a}
\end{gathered}
$$

If $a>0$, when $\delta \rightarrow 0, \delta^{a} \rightarrow 0$, (47) is convergent. If $a<0$, when $\delta \rightarrow 0, \delta^{a} \rightarrow \infty$, (47) is divergent.

So, the last result of (44) should be

$$
\int_{\Omega}^{(-z)^{s-1}} \frac{e^{z}-1}{d z}=Q(\delta)
$$

If $a<1$, when $\delta \rightarrow 0$, we have $Q(\delta) \rightarrow \infty$. Similar to the summation form of series shown in (1), the integral form of Riemann Zeta function is still divergent when $\operatorname{Re}(s)=a<1$. Therefore, it is meaningless to discuss the zeros of integral form of Riemann Zeta function at point $a=1 / 2<1$.

Some authors also estimated and make approximate calculations on the situation $a>1$ and came to the same conclusion that (17) could be ignored, but unfortunately no further calculations were carried out $[2,10]$. In fact, if they also did in-depth calculations for the situation $a<1$, they would have reached the same conclusion that the item can not be neglected.

\subsection{The Numerical Calculation of Divergent Item}

In order to have more clear understanding of divergence, let's take numerical calculation. We have from (15) 


$$
\begin{aligned}
I(s)= & \int_{\infty}^{\delta} \frac{(-x)^{s-1}}{e^{x}-1} d x+\int_{\Omega} \frac{(-z)^{s-1}}{e^{z}-1} d z+\int_{\delta}^{\infty} \frac{(-x)^{s-1}}{e^{x}-1} d x \\
& =\left(e^{i \pi s}-e^{-i \pi s}\right) \int_{\delta}^{\infty} \frac{x^{s-1}}{e^{x}-1} d x+Q(\delta) \\
& =2 i \sin (\pi s) \int_{\delta}^{\infty} \frac{x^{s-1}}{e^{x}-1} d x+Q(\delta)
\end{aligned}
$$

By considering (14) and (49), we get

$$
\int_{\delta}^{\infty} \frac{x^{s-1}}{e^{x}-1} d x=\frac{1}{2 i \sin (\pi s)}\left[\int_{L} \frac{(-z)^{s-1}}{e^{z}-1} d z-Q(\delta)\right]
$$

If $\delta \neq 0$, we have

$$
\begin{aligned}
& \zeta(s) \Gamma(s)=\int_{0}^{\infty} \frac{x^{s-1}}{e^{x}-1} d x \neq \int_{\delta \neq 0}^{\infty} \frac{x^{s-1}}{e^{x}-1} d x \\
& =\frac{1}{2 i \sin (\pi s)}\left[\int_{L} \frac{(-z)^{s-1}}{e^{z}-1} d z-Q(\delta \neq 0)\right]
\end{aligned}
$$

On the other hand, on the real axis of complex plane with $b=0$, according to (46), we have approximately

$$
Q(\delta)=\frac{\delta^{a-1}}{a-1} \cos (\pi(a-1))
$$

If $a>1$, when $\delta \rightarrow 0$, we have $Q(\delta) \rightarrow 0$. This is just the result of Riemann's original paper

$$
\zeta(s) \Gamma(s)=\int_{0}^{\infty} \frac{x^{s-1}}{e^{x}-1} d x=\frac{1}{2 i \sin (\pi s)} \int_{L} \frac{(-z)^{s-1}}{e^{z}-1} d z
$$

If $a<1$, for example, to take $a=3 / 4$, we have from (52)

$$
Q(\delta)=-\frac{\delta^{-1 / 4}}{1 / 4} \cos \left(-\frac{\pi}{4}\right)=-\frac{2 \sqrt{2}}{\delta^{1 / 4}}
$$

When $\delta \neq 0$, taking $\delta=10^{-4}$, we have $Q(\delta)=-2 \sqrt{2} \times 10$, (50) becomes

$$
\begin{aligned}
& \int_{10^{-4}}^{\infty} \frac{x^{s-1}}{e^{x}-1} d x=\frac{1}{2 i \sin (\pi s)} \\
& \times\left[\int_{L}^{(-z)^{s-1}} \frac{\left(e^{z}-1\right.}{d z}+2 \sqrt{2} \times 10\right]
\end{aligned}
$$

There is an additional item $2 \sqrt{2} \times 10$ in (55). Taking $\delta=10^{-40}$, we have $Q(\delta)=-2 \sqrt{2} \times 10^{10},(50)$ becomes

$$
\int_{10^{-40}}^{\infty} \frac{x^{s-1}}{e^{x}-1} d x=\frac{1}{2 i \sin (\pi s)}
$$

$$
\times\left[\int_{L} \frac{(-z)^{s-1}}{e^{z}-1} d z+2 \sqrt{2} \times 10^{10}\right]
$$

The additional item has the magnitude of $10^{10}$. In fact, for $\operatorname{Re}(s)<1$, as long as $\delta$ is small enough, $|-Q(\delta)|$ can be great enough with

$$
|-Q(\delta)|>>\int_{L} \frac{(-z)^{s-1}}{e^{z}-1} d z
$$

so that we have

$$
\zeta(s) \Gamma(s)=\int_{0}^{\infty} \frac{x^{s-1}}{e^{x}-1} d x \neq \frac{1}{2 i \sin (\pi s)} \int_{L} \frac{(-z)^{s-1}}{e^{z}-1} d z
$$

If taking $\delta \rightarrow 0$, when $\operatorname{Re}(s)<1$, we have $Q(\delta) \rightarrow \infty$ and

$$
\begin{aligned}
& \zeta(s) \Gamma(s)=\int_{0}^{\infty} \frac{x^{s-1}}{e^{x}-1} d x \\
& =\frac{1}{2 i \sin (\pi s)}\left[\int_{L} \frac{(-z)^{s-1}}{e^{z}-1} d z+\infty\right]
\end{aligned}
$$

In this case, the integral form of Riemann Zeta function has not changed the divergence of its summation form and is actually meaningless.

\subsection{The Applicable Condition of Formula (12)}

The reasons to cause the inconsistency and divergence of Zeta function equation are discussed below. According to the definition of $\Gamma(s)$, when $\operatorname{Re}(s)>0$, the integral of (9) is limited at its lower limit $x=0$. (12) is used when Riemann deduced (13). It noted that the applicable condition of (12) is $0<x \leq \infty$. This condition can be found in any mathematics handbook [8] (Let $x^{\prime}=e^{-x},\left|x^{\prime}\right|>1$ ). At point $x=0$, two sides of (12) become infinite and meaningless with

or

$$
\begin{gathered}
\sum_{n=1}^{\infty} e^{-n x}=e^{-x}+e^{-2 x}+e^{-3 x}+\cdots=\frac{1}{e^{x}-1} \rightarrow \infty \\
1+1+1+1+\cdots=\frac{1}{0} \rightarrow \infty
\end{gathered}
$$

Besides, contradictory result would be caused by (12) when $x=0$. For example, when $0<x \leq \infty$, according to (12), we have

$$
\begin{aligned}
& \sum_{n=1}^{\infty} e^{-n x}=e^{-x}+e^{-2 x}+e^{-3 x}+\cdots+1000>\frac{1}{e^{x}-1} \\
& \sum_{n=1}^{\infty} e^{-n x}=e^{-x}+e^{-2 x}+e^{-3 x}+\cdots-1000<\frac{1}{e^{x}-1}
\end{aligned}
$$


If let $x=0,(61)$ and (62) becomes

$$
\infty+1000=\infty>\infty \text { and } \infty-1000=\infty<\infty
$$

These results are absurd, so (12) can not be used at point $x=0$ and (13) can not hold. In Riemann's original paper, (12) and its applicable condition had not been mentioned. Besides (12) is improper at $x=0$, we seems unable to find other reason to cause the inconsistency in (30) and the infinite in (59). Meanwhile, just by using (12) and introducing the Residue theorem, the summation form of $\zeta(1-s)$ was obtained which caused contradiction with $\zeta(s)$ on the two sides of Zeta function equation.

\section{The Formula of Jacobi Function is Improper and Unusable}

Riemann proved that the Zeta function equation had the symmetry $\xi(s)=\xi(1-s)$ in his original paper. The proof was not clear enough. We give the detail below and prove that the symmetry does not exist due to the incorrect using of the formula of Jacobi function. The following Gama function was used in the deduction which was different from $(9)[1,2]$.

$$
\Gamma\left(\frac{\mathrm{s}}{2}\right)=\int_{0}^{\infty} e^{-x} x^{s / 2-1} d x
$$

Let $x \rightarrow n^{2} \pi x$ in (64), Riemann obtained

$$
\begin{gathered}
\Gamma\left(\frac{\mathrm{s}}{2}\right)=\int_{0}^{\infty} e^{-n^{2} \pi x} n^{s} \pi^{s / 2} x^{s / 2-1} d x \\
\left(\sum_{n=1}^{\infty} n^{-s}\right) \pi^{-s / 2} \Gamma\left(\frac{\mathrm{s}}{2}\right)=\int_{0}^{\infty} x^{s / 2-1}\left(\sum_{n=1}^{\infty} e^{-n^{2} \pi x}\right) d x
\end{gathered}
$$

Set

$$
\psi(x)=\sum_{n=1}^{\infty} e^{-n^{2} \pi x}
$$

By means of the definition (1), (66) was written as

$$
\begin{aligned}
& \zeta(s) \pi^{-s / 2} \Gamma\left(\frac{\mathrm{s}}{2}\right)=\int_{0}^{\infty} x^{s / 2-1} \psi(x) d x \\
= & \int_{0}^{1} x^{s / 2-1} \psi(x) d x+\int_{1}^{\infty} x^{s / 2-1} \psi(x) d x
\end{aligned}
$$

In order to calculate (68), the Jacobi's function was introduced with

$$
\theta(x)=\sum_{n=-\infty}^{\infty} e^{-n^{2} \pi x}=1+2 \sum_{n=1}^{\infty} e^{-n^{2} \pi x}=1+2 \psi(x)
$$

The Jacobi's function satisfied following equation $[7,11]$

$$
\theta(x)=\sqrt{x} \theta\left(\frac{1}{x}\right) \quad x>0
$$

So (69) can be written as

$$
\begin{gathered}
1+2 \psi(x)=\theta(x)=\frac{1}{\sqrt{x}} \theta\left(\frac{1}{x}\right) \\
=\frac{1}{\sqrt{x}}\left[2 \psi\left(\frac{1}{x}\right)+1\right]
\end{gathered}
$$

i.e.

$$
\psi(x)=\frac{1}{2}\left\{\frac{1}{\sqrt{x}}\left[2 \psi\left(\frac{1}{x}\right)+1\right]-1\right\}
$$

By means of $(72)$, we obtain $[1,11]$

$$
\begin{gathered}
\int_{0}^{1} x^{s / 2-1} \psi(x) d x=\frac{1}{2} \int_{0}^{1} x^{s / 2-1}\left\{\frac{1}{\sqrt{x}}\left[2 \psi\left(\frac{1}{x}\right)+1\right]-1\right\} d x \\
=\int_{0}^{1} x^{s / 2-3 / 2} \psi\left(\frac{1}{x}\right) d x+\frac{1}{2} \int_{0}^{1}\left(x^{-s / 2-3 / 2}-x^{s / 2-1}\right) d x \\
\frac{1}{2} \int_{0}^{1}\left(x^{s / 2-3 / 2}-x^{s / 2-1}\right) d x=\frac{1}{s(s-1)}
\end{gathered}
$$

Let $x=1 / y$ in (73), we get

$$
\begin{gathered}
\int_{0}^{1} x^{s / 2-3 / 2} \psi\left(\frac{1}{x}\right) d x=\int_{\infty}^{1} y^{-s / 2+3 / 2} \psi(y)\left(-y^{-2}\right) d y \\
=\int_{1}^{\infty} y^{-(s+1) / 2} \psi(y) d y
\end{gathered}
$$

Let $y \rightarrow x$ on the right side of (75) and considering (74), (73) becomes

$$
\int_{0}^{1} x^{s / 2-1} \psi(x) d x=\int_{1}^{\infty} x^{-(s+1) / 2} \psi(x) d x+\frac{1}{s(s-1)}
$$

Thus, (68) can be written as

$$
\begin{gathered}
\zeta(s) \pi^{-s / 2} \Gamma\left(\frac{\mathrm{s}}{2}\right) \\
=\int_{1}^{\infty} \psi(x)\left(x^{-(s+1) / 2}+x^{s / 2-1}\right) d x+\frac{1}{s(s-1)}
\end{gathered}
$$

The right side of (77) is unchanged under the replace $s \rightarrow 1-s$, so its left side should be invariable too under 
$s \rightarrow 1-s$. Set $[12,13]$

$$
\Phi(s)=\pi^{-s / 2} \Gamma\left(\frac{\mathrm{s}}{2}\right) \zeta(s)
$$

(77) indicates the existence of symmetry

$$
\Phi(s)=\Phi(1-s)=\pi^{-(1-s) / 2} \Gamma\left(\frac{1-s}{2}\right) \zeta(1-s)
$$

From (78) and (79), we obtain

$$
\pi^{-s / 2} \Gamma\left(\frac{\mathrm{s}}{2}\right) \zeta(s)=\pi^{-(1-s) / 2} \Gamma\left(\frac{1-s}{2}\right) \zeta(1-s)
$$

By means of the complemented formula of Gama function

$$
\Gamma\left(\frac{s}{2}\right) \Gamma\left(1-\frac{s}{2}\right)=\frac{\pi}{\sin (s \pi / 2)}
$$

(80) can be written as

$$
\zeta(s)=\pi^{s-3 / 2} \sin \frac{s \pi}{2} \Gamma\left(1-\frac{s}{2}\right) \Gamma\left(\frac{1-s}{2}\right) \zeta(1-s)
$$

On the other hand, based on (9), Riemann obtained (13). By extending (13) to the field of complex plane and using the residue theorem, Riemann deduced the function equation (5). If these two methods are consistent, (82) and (5) should be consistent. We should have

$$
\pi^{\mathrm{s}-3 / 2} \Gamma\left(1-\frac{s}{2}\right) \Gamma\left(\frac{1-s}{2}\right)=2(2 \pi)^{s-1} \Gamma(1-s)
$$

or

$$
\Gamma\left(1-\frac{s}{2}\right) \Gamma\left(\frac{1-s}{2}\right)=2^{s} \pi^{1 / 2} \Gamma(1-s)
$$

On the other hand, we have the Legendre double formula of Gama function

$$
\Gamma\left(s+\frac{1}{2}\right) \Gamma(s)=2^{1-2 s} \pi^{1 / 2} \Gamma(2 s)
$$

Let $s \rightarrow(1-s) / 2$ in (85), we get (84) and prove (83), so the results of two methods are the same. But if the neglected item in (5) which is infinite when $\operatorname{Re}(s)<1$ is considered, both calculations are not consistent again.

Meanwhile, this deduction had a serious problem. The applicable condition of Jacobi's equation (70) is $x>0$. If $x=0$, we have $1 / x \rightarrow \infty$, (70) becomes meaningless. Riemann did not mention this condition in his original paper. Because the lower limits of integrals (73) and (74) are $x=0$, the Jacobi's equation (70) can not be used, (77) can not hold. The symmetry of (79) does not exist.

\section{Discussion on the Zero's Calculation of Zeta Function Equation}

By means of manual and numerical calculations, a great number of zeros were founded up to now (about one thousand billion) $[14,15]$. All of them were regarded to locate on the straight line of $\operatorname{Re}(s)=1 / 2$. However, they are not the real zeros of strict Zeta function. Let's discuss this problem.

1. For a common complex function $f(z)=f(x+i y)$, its real part and imaginary part can always be separated and written in following form

$$
f(z)=u(x, y)+i v(x, y)
$$

If $f(z)$ is analytic one, its real part and imaginary part are independent. They should satisfy the following Cauchy-Riemann equation [9]

$$
\frac{\partial u}{\partial x}=\frac{\partial v}{\partial y} \quad \frac{\partial u}{\partial y}=-\frac{\partial v}{\partial x}
$$

Riemann Zeta function is an analytic one, so (7) can also be written as;

$$
\xi(s)=u(a, b)+i v(a, b)
$$

$u(a, b)$ and $v(a, b)$ should satisfy (87). $\xi(s)=0$ means that $u(a, b)$ and $v(a, b)$ are equal to zeros simultaneously. However, in the zero's calculation of Zeta function, approximate method was used so that the Cauchy-Riemann equation (87) was not satisfied.

2. For example, let $s=1 / 2+i b$, (7) was written as $[14,16]$

$$
\begin{gathered}
\xi(1 / 2+i b)=\left[e^{R e \ln \Gamma(s / 2)} \pi^{-1 / 4} \frac{-b^{2}-1 / 4}{2}\right] \\
\times\left[e^{i I m \ln \ln \Gamma(s / 2)} \pi^{-i b / 4} \zeta(1 / 2+i b)\right]
\end{gathered}
$$

The practical process to calculate zero was to calculate the changes of signs in (89). If the sign of Zeta function changed from positive to negative or from negative to positive, a zero was considered to be found. Because the item in the first bracket was always negative, it could be neglected. We only need to calculate the item in the second bracket. Let

$$
Z(b)=e^{i \operatorname{Im} \ln \ln \Gamma(s / 2)} \pi^{-i b / 4} \zeta(1 / 2+i b)
$$

By developing $Z(b)$ into the progressive form, the Riemann-Siegal formula was obtained [14]

$$
\mathrm{Z}(b)=2 \sum_{n<\sqrt{t / 2 \pi}} n^{-1 / 2} \cos (\theta(b)-b \ln n)+R(b)
$$

The zeros calculation was based on (91), in which $\theta(b)$ and $R(b)$ were very complex functions. By taking the different 
orders of $Z(b)$, different zeros were found. For example, the first item of (91) was $Z(b)=2 \cos \theta(t)$ and the first zero was at $b \approx 14.5$. Then, by considering the revised factor $R(b)$, the value was revised into $b \approx 14.1345$, and so on.

It is obvious that original complex Zeta function had changed into real function in (91). $Z(b)$ was neither real part nor imaginary part of original Zeta function. The restriction of the Cauchy-Riemann equation did not exist again. Because (91) contains trigonometric function, it had infinite $b$ to satisfy $Z(b)=0$. However, they were not real zeros of the strict Zeta function.

3. It assumed $\operatorname{Re}(s)=1 / 2$ in advance in all computer calculations about the zeros of Zeta function. Because this is a precondition, we can not say that the calculations of computer have proved that all zeros are located on the line of $\operatorname{Re}(s)=1 / 2$. What we can say is that there are great numbers of zeros on the line of $\operatorname{Re}(s)=1 / 2$.

4. In fact, according to (1) and (28), $\zeta(s)$ and $\zeta(1-s)$ are already series. We can discuss their zeros directly. It is unnecessary for us to develop them into series again. The Riemann-Siegal formula (91) not only causes errors, but also perplexes the problem.

\section{Conclusion}

The Riemann hypothesis was one of 23 mathematical problems presented by Hilbert at the world mathematical congress in 2000. It was not solved in the 20th century and was chosen by Clay Institute of Mathematics as one of the eight millennium mathematical problems.

The Riemann hypothesis is so famous, also due to the fact that it has become the important basis of modern prime number distribution theory. Because of Riemann's work, the traditional prime distribution theory was extended from the real domain to the complex domain. According to statistics, nearly a thousand theorems had been proposed based on the premise of assuming the Riemann hypothesis to be correct so far. We can see the depth of its influence on modern mathematics.

Since the Riemann hypothesis had not been proved for a long time, some mathematicians believed that it was not true. But most mathematicians believed it to be true, and they wanted it to be true. However, this paper gives third result. The Riemann hypothesis is proved to be meaningless, which is unexpected by most people.

The problem lied in Riemann's original paper of 1859. In the Riemann's deduction, two formulas were improperly used without considering their applicable conditions which caused the inconsistency of the Zeta function equation. Meanwhile, an integral term around the origin point of coordinate system was omitted. This item was infinite when $\operatorname{Re}(s)<1$, so the integral form of Zeta function had not changed the divergence of its series summation form.

Because the integral form of Zeta function and its function equation are not tenable generally when $\operatorname{Re}(s)<1$, the
Riemann hypothesis becomes meaningless. Mathematicians should consider whether it makes sense to research the distribution of prime numbers based on the Riemann Zeta function equation again. In other words, we should consider whether or not the study on prime number distribution should return to the traditional model in the real number domain.

\section{References}

[1] Riemann G. F. B., Uber die Anzabl der Primahlem unter einer gegebenen Grosse, Monatsberichte der Berliner Akademine, $1859,2,671-680$.

[2] Bent E. Petersen, Riemann Zeta Function, https://pan. baidu.com/s/1geQsZxL.

[3] Neukirch, J., Algebraic Number Theory, 1999, Springer, Berlin, Heidelberg (The original German edition was published in 1992 under the title Algebraische Zahlentheorie).

[4] Iwaniec H., Lectures on the Riemann Zeta Function, American Mathematical Society, 2014, Providence.

[5] Bender, C. M., Brody, D. C., M"uller, M. P., Hamiltonian for the zeros of the riemann zeta function, Physical Review Letters, 2017, 118 (13), 130201.

[6] Lagarias, J. C. An elementary problem equivalent to the Riemann hypothesis, The American Mathematical Monthly, 2002, 109 (5), 534-543.

[7] Jacobi, C. G. J., Fundamenta Nova Theoriae Functionum Ellipticarum, Regiomonti, Borntraeger, Konigsberg, 1829, (Reprinted by Cambridge University Press, 2012).

[8] Mathematics Handbook, Scientific Publishing House, 1980, p. 144.

[9] Guo Dunreng, The Methods of Mathematics and Physics, Education publishing House, 1965, p. 109.

[10] Felix Rubin, Riemann's First Proof of the Analytic Continuation of Zeta function, http://www2.math. ethz.ch/edu cation/bachelor/seminars/ws0607/modular-forms/Riemanns_fi rst_proof.pdf.

[11] Gerald Tenenbaum, Michel Mendes France, Les Nombres, Premiers, Presses Universitaires de France, 1997, 44-52.

[12] Gelbart, S., Miller, S., Riemann's zeta function and beyond, Bulletin of the American Mathematical Society, 2004, 41 (1), 59-112.

[13] Jessen, B., Wintner, A., Distribution functions and the Riemann zeta function, Transactions of the American Mathematical Society, 1935, 38 (1), 48-88.

[14] Ru Changhai, The Riemann Hypothesis, Qianghua University Publishing Company, 2016, p. 61, 52, 192.

[15] Gourdon X., The $10^{\wedge}(13)$ first zeros of the Riemann Zeta function, and zeros computation at very large height, 2004, http://pdfs.semanticscholar.org/6eff/62ff5d98e8ad2ad8757c0f af4bac87546f27. pdf.

[16] Katz N M, Sarnak P. Zeroes of zeta functions and symmetry [J]. AMS, 1999, 36 (1) 1-26. 